\title{
Human Papillomavirus-Associated Head and Neck Cancer
}

\author{
Juan C. Nogues, BS, Scott Fassas, MD, Collin Mulcaby, MD, and \\ Philip E. Zapanta, MD
}

Oropharyngeal squamous cell carcinoma (OPSCC) has historically been attributable to tobacco and alcohol exposure and saw a decline in incidence after societal norms shifted away from smoking. In recent decades, this disease has had a re-emergence due to human papillomavirus (HPV) infection, now surpassing cervical cancer as the number 1 cause of HPV-related cancer in the United States. HPVpositive OPSCC differs from HPV-negative disease in epidemiology, prognosis, treatment, and prevention. Additionally, there is a deficit in awareness of the causal relationship between HPV and OPSCC. This, coupled with low vaccination rates, puts primary care providers in a unique position to play a vital role in prevention and early diagnosis. In this review, we highlight the epidemiology, screening, patient presentation, diagnosis, prognosis, and prevention of HPV-positive OPSCC, with a focus on the primary care provider's role. (J Am Board Fam Med 2021;34:832-837.)

Keywords: Immunization, Otolaryngology, Papillomavirus Vaccines, Patient Care Team, Primary Health Care, Sexually Transmitted Diseases, Squamous Cell Carcinoma of Head and Neck

\section{Introduction}

Oropharyngeal squamous cell carcinoma (OPSCC) has historically been attributable to tobacco exposure, seeing a decline in incidence in the 1980s (Figure 1) $)^{1}$ after societal norms shifted away from smoking. However, in recent decades this disease has had a reemergence due to human papillomavirus (HPV) infection, now surpassing cervical cancer as the number 1 cause of HPV-related cancer in the United States. ${ }^{2}$ Compared with HPV-negative OPSCC, the disease course of HPV-positive OPSCC portends a more favorable prognosis, with significantly increased progression free survival. ${ }^{3}$ This is particularly true when appropriate therapy is provided early, highlighting the importance of early detection and treatment to reduce the morbidity associated with late-stage interventions. Although treatment interventions are improving,

This article was externally peer reviewed.

Submitted 11 November 2020; revised 3 March 2021; accepted 11 March 2021.

From Division of Otolaryngology-Head and Neck Surgery, George Washington University, Washington, DC (JCN, SF, CM, PEZ).

Funding: None.

Conflict of interest: None.

Corresponding author: Juan C. Nogues, BS, 3536 NW 17 ST, Miami, FL 33125 (E-mail: nogues@gwmail.gwu.edu). the majority of these cases are attributable to HPV 16, 18, 31, and 33; all infections preventable by vaccination.

This puts primary care providers (PCPs) in a unique position to play a vital role in prevention, screening, and early diagnosis. In this review, we highlight the epidemiology, screening, patient presentation, diagnosis, prognosis, and prevention of HPV-positive OPSCC, with a PCP-focused approach.

\section{Epidemiology}

OPSCC has historically been a disease affecting older male smokers. As the rates of tobacco consumption have declined in the United States, there has been a decreased incidence of many head and neck cancers. HPV-related head and neck cancers, however, have not followed this overall trend, with multiple sources demonstrating a rise in incidence over the last 2 decades. ${ }^{4-6}$ Specifically, the percentage of HPV-related cancers has jumped from $41 \%$, before 2000, to $72 \%$ between 2005 and 2009 (Figure 2). ${ }^{7}$ While previous literature estimated the incidence of HPV-related OPSCC would surpass HPV-related cervical cancer by $2020,{ }^{8,9}$ data collected by the Centers for Disease Control and 
Figure 1. SEER Age-Adjusted Trends in Oral Cavity and Pharynx Cancer, 1975 to $2017 .{ }^{1}$ Results from cancer incidence data from population-based cancer registries covering approximately $34.6 \%$ of the US population.

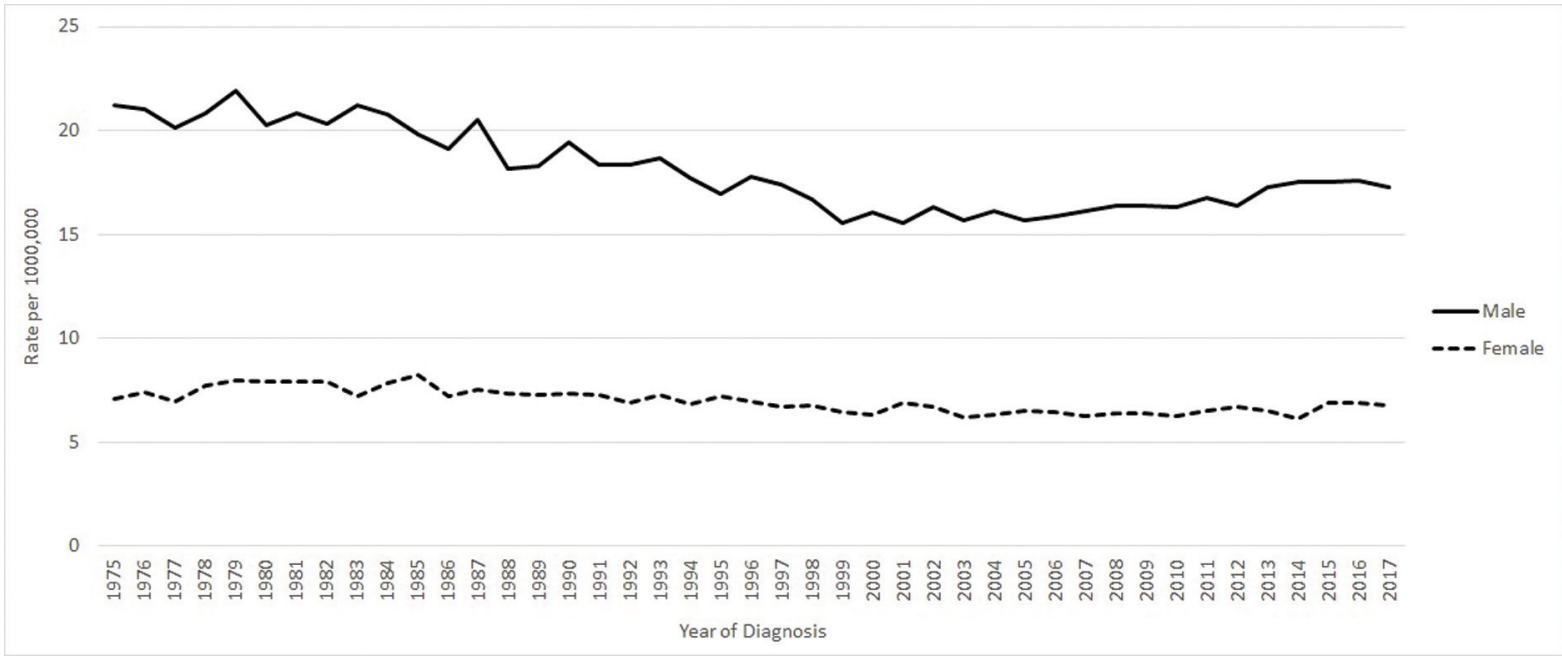

Prevention (CDC) have suggested that the total incidence has already surpassed HPV-related cervical cancer well before this time frame. ${ }^{2}$

While marijuana exposure has been shown to be an independent risk factor for the development of $\mathrm{HPV}$-positive head and neck cancer, ${ }^{10}$ the primary reason for the increase in OPSCC is related to an overall rise in high-risk sexual practices. More than $90 \%$ of oral HPV infections are related to sexual activity, with oral sex being the largest predisposing factor. ${ }^{11,12}$ Furthermore, patients impacted by
HPV-positive OPSCC are generally younger than their HPV-negative counterparts and overwhelmingly white males-typically between the ages of 40 and $55 .{ }^{13}$

\section{Screening and Presentation}

Unlike cervical cancer, which uses Papanicolaou cytology screening to assess precancerous lesions of the cervix, there are currently no practical screening tools available for HPV-positive OPSCC screening. ${ }^{12}$ Several techniques are under investigation,

Figure 2. Human Papillomavirus (HPV) Prevalence Among Patients With Oropharyngeal Cancer as Reported in A Systematic Review and Meta-Analysis of Studies Published Between 1966 and $2010 .^{7}$

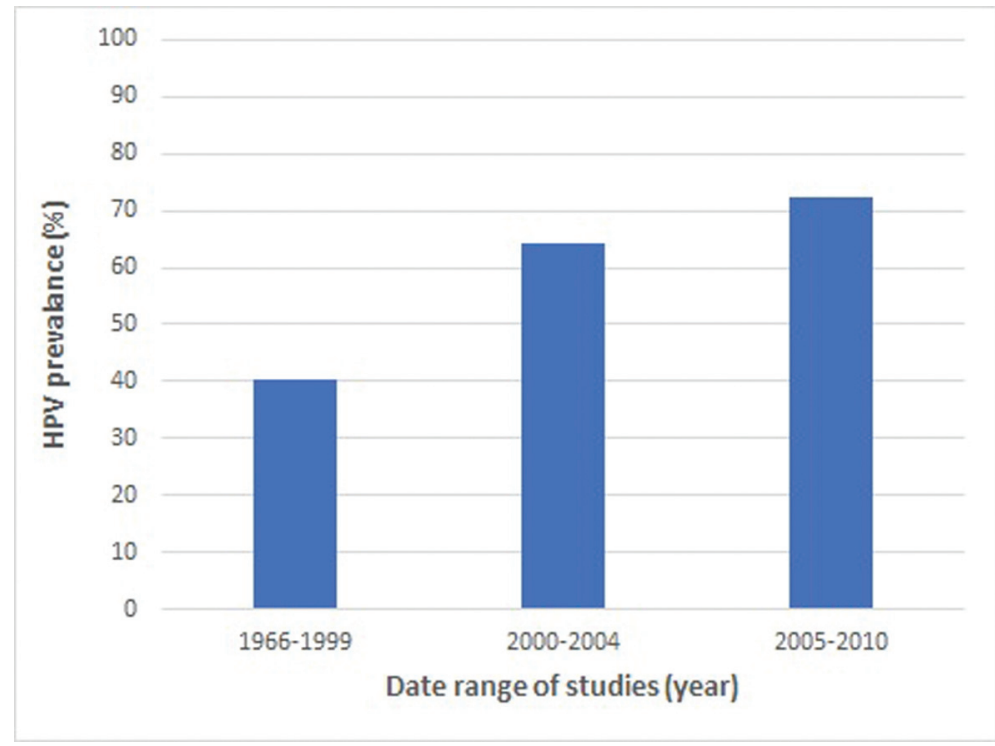


but each has limitations that preclude its use for population-level screening (Table 1). Furthermore, validated sexual history screening questionnaires have not been developed to capture patients most at risk.

Without a validated screening test or questionnaire to detect patients with or most at risk for $\mathrm{HPV}$-positive OPSCC, it is imperative that firstline health care providers (PCPs and dentists) remain cognizant of the current epidemiologic factors discussed above. This information, coupled with knowledge and awareness of common clinical presentations, can be greatly beneficial to patients. A recent retrospective analysis of 207 patients with OPSCC found that patients with HPV-positive OPSCC were most likely to present with a neck mass $(56 \%)$, sore throat $(11 \%)$, or oral mass (11\%). ${ }^{14}$ Furthermore most HPV-positive patients in this study presented at a tumor, node, metastasis stage of IVa (57\%), with the tonsil (60\%) and base of tongue $(40 \%)$ being the most common primary tumor site. ${ }^{14}$

If a patient presents to the office with a history suspicious for OPSCC, it is recommended that providers conduct a formal head and neck examination that includes inspection of the oral cavity, palatine tonsils, base of tongue, and cervical lymph nodes. An external light source and tongue blade are important for optimum visualization of the oral cavity and oropharynx for asymmetries or lesions. A mirror may be used to inspect the vallecula. Thorough palpation of the tonsillar fossae and tongue base can identify induration, ulceration, or swelling. ${ }^{15}$

\section{Prevention}

The majority of HPV-positive OPSCCs are caused by HPV 16 with minor contributions from 18, 31, and 33. In 2006, the first HPV vaccine, Gardasil 4, was approved for prevention of cervical cancer and covered HPV 6, 11, 16, and 18. The latest vaccine, Gardasil 9, covers HPV strains 6, 11, 16, 18, 31, 33, 45,52 , and 58 and therefore protects against the most carcinogenic strains of HPV. It has gained US Food and Drug Administration (FDA) approval for prevention of cervical, vulvar, vaginal, anal, oropharyngeal, and other head and neck cancers ${ }^{16}$ and is administered in either 2 or 3 doses depending on the age of the patient at the time of the first dose (Table 2). The primary target group for the vaccine is children over the age of 9 , ideally before the onset of sexual activity; however, Gardasil 9 has been FDA approved for both men and women up to the age of $45 .^{17}$ Despite this expanded coverage, the CDC does not currently recommend routine HPV vaccination for patients above the age of 26 but rather shared clinical decision-making on a case-by-case basis. ${ }^{18,19}$ Because of this, insurance

\section{Table 1. Proposed HPV Screening Tools ${ }^{12}$}

\begin{tabular}{|c|c|c|}
\hline Screening Tool & Concept & Limitation \\
\hline Oral HPV screening & $\begin{array}{l}\text { Sample patient's saliva and test for high-risk } \\
\text { HPV viral DNA }\end{array}$ & $\begin{array}{l}\text { Although this can detect the presence of HPV DNA, the } \\
\text { majority of individuals will go on to clear the virus } \\
\text { without progressing to carcinoma }\end{array}$ \\
\hline HPV serology & $\begin{array}{l}\text { Asses serum levels of antibodies to high-risk } \\
\text { HPV strands }\end{array}$ & $\begin{array}{l}\text { Serum antibody levels represent the cumulative exposure } \\
\text { to HPV but are not specific to exposure at a particular } \\
\text { anatomic site (oropharynx, cervix, etc.) and do not } \\
\text { reflect expression of HPV-related oncoproteins E6 and } \\
\text { E7, which are necessary for carcinogenesis }\end{array}$ \\
\hline Transcervical ultrasound & $\begin{array}{l}\text { Ultrasound of the head and neck can be } \\
\text { used to assess individuals with neck } \\
\text { masses or those who are found to be at } \\
\text { high risk via other screening methods. It } \\
\text { may help detect small tumors in earlier } \\
\text { stages and improve morbidity }\end{array}$ & $\begin{array}{l}\text { Although ultrasound is relatively inexpensive and can be } \\
\text { done quickly in the office, it is not practical for } \\
\text { universal screening and relies on either the symptom } \\
\text { of a neck mass or other screening method to prescreen } \\
\text { high-risk individuals }\end{array}$ \\
\hline Mucosal imaging & $\begin{array}{l}\text { Direct visualization of subclinical lesions via } \\
\text { endoscopy }\end{array}$ & $\begin{array}{l}\text { There are no identifiable premalignant lesions, and as } \\
\text { with the limitations of ultrasound, there needs to be a } \\
\text { prescreening method to identify high-risk individuals } \\
\text { before subjecting them to this more invasive } \\
\text { procedure. Additionally, many of these tumors arise } \\
\text { from tonsillar crypts and cannot be easily identified on } \\
\text { surface-level examination }\end{array}$ \\
\hline
\end{tabular}

HPV, Human papillomavirus; DNA, Deoxyribonucleic acid. 
Table 2. Dosing Schedule of Gardasil 9 Vaccine as Recommended by Manufacturer ${ }^{16}$

\begin{tabular}{lcc}
\hline Age & $\begin{array}{c}\text { Number of } \\
\text { Doses }\end{array}$ & Dosing Interval \\
\hline 9 to 14 & 2 or 3 & Second dose 6-12 months after \\
the first & 0,2 months, 6 months \\
15 to 45 & 3 & 0,2 months, 6 months \\
\hline
\end{tabular}

may not always cover the vaccine in this extended age group.

Despite the wide availability of HPV vaccines in the United States, many providers are unaware of the link between HPV and OPSCC. A 2017 study reported that only $16 \%$ of pediatricians were aware of the link between OPSCC and HPV, and only $46 \%$ had knowledge that HPV-related oropharyngeal cancer incidence was increasing in the United States. ${ }^{20}$ What is more alarming is that a 2019 national vaccination study found that among adolescents between 13 and 17 years old, only 54\% were up to date with the recommended HPV vaccination series. ${ }^{21}$ Males (52\%) were shown to have a slightly lower vaccination rate than females (57\%).

The lack of awareness and low vaccination rate highlights the paramount role that PCPs play in disease prevention. While the vaccine is targeted for virginal adolescents, a recent study of men between the ages of 27 and 45 found that the immune response in this population was comparable to that of younger men ages 16 to $26 .^{22}$ Armed with this information, PCPs can increase HPV vaccination rates and greatly reduce the morbidity and mortality associated with OPSCC.

In addition, oro-genital sex is the most important risk factor for developing $\mathrm{HPV}$-positive OPSCC. While studies have shown that properly utilized barrier methods of protection (condoms and dental dams) can lower the risk of contracting $\mathrm{HPV},{ }^{23}$ in practice, they have poor adoption and user compliance for oral sex, especially among young adults. ${ }^{24}$ Thus, although it is prudent to suggest barrier use for patients who are receptive, it should not serve as an alternative to vaccination, especially for high-risk individuals with multiple sexual partners.

Lastly, smoking is a well-known risk factor for HPV-negative OPSCC but may also be associated with increased risk of HPV-positive OPSCC. A recent study demonstrated that smoking prolongs
HPV infections in the oral cavity, ${ }^{25}$ suggesting it could play a role in the pathogenesis of HPVrelated OPSCC. This provides more evidence to support smoking cessation for patients, especially those at high risk or who have not been vaccinated.

\section{Diagnosis}

While it is important to know the common clinical presentation, a high index of suspicion is necessary when patients have presentations concerning for a neck mass. If a lesion is identified, suspected but not visualized, or the patient endorses troublesome symptoms, a low threshold for referral to an otolaryngologist for further workup is necessary. Workup includes imaging and visualization of primary sites via endoscopy, although OPSCC can only be diagnosed via biopsy of a primary or metastatic lesion. Following the biopsy, tumor samples will undergo either polymerase chain reaction amplification of HPV DNA or in situ hybridization to determine if it is HPV-positive or HPV-negative.

\section{Treatment}

Trends in the treatment of patients with OPSCC are constantly evolving. Historically, large open resections were the predominant treatment approach for these patients. Due to severe functional morbidity and high complication rates associated with these invasive procedures, chemoradiation (CRT) became the preferred treatment option in the 1990s. ${ }^{26}$ CRT provides equivalent overall survival for patients with OPSCC with less functional morbidity when compared with open surgery. Unfortunately, as CRT became more widely adopted, it was clear that it had its own toxicity profile, including dysphagia, osteoradionecrosis, mucositis, and xerostomia, all which have profound effects on the quality of life for cancer survivors. ${ }^{27}$ With the rise in HPV-related OPSCC, and a much younger population being affected, it was imperative to consider the long-term consequences of the treatment regimen. This in part is why treatment again shifted toward less invasive surgical approaches such as transoral robotic surgery (TORS). TORS has been shown to have equivalent outcomes as CRT, and it may allow for improved long-term outcomes from CRT de-escalation. ${ }^{28}$ Currently, there is literature to support improved quality of life in patients treated with TORS, ${ }^{29,30}$ 
although the subject at the moment is controversial. Recently the ORATOR trial, a phase 2 randomized study demonstrated that patients undergoing TORS actually had worse swallowing-related quality of life scores 1 year after treatment, compared with the radiotherapy-treated group. ${ }^{31}$

\section{Follow-up}

Follow-up for these patients is complex, often requiring multiple specialists to treat their disease such as medical oncologists, head and neck radiation oncologists, otolaryngologists, speech language pathologists, and nutritionists. The PCP is not only essential in ensuring that the patient is following up appropriately with specialist services but is also vital in managing any other illnesses or comorbidities that would significantly impact the prognosis. Follow-up with otolaryngology is generally extensive, and patients are seen for many years following treatment for tumor surveillance.

\section{Conclusions}

HPV-positive OPSCC is the most common HPVrelated malignancy in the United States and affects a different demographic of individuals than HPVnegative OPSCC. PCPs are crucial in primary prevention of disease through vaccination and can dramatically improve patient prognosis through early detection. By increasing awareness of the distinctions between $\mathrm{HPV}$-negative and $\mathrm{HPV}$-positive OPSCC among PCPs, we could decrease the burden of illness among the population.

To see this article online, please go to: bttp://jabfm.org/content/ 34/4/832.full.

\section{References}

1. National Cancer Institute Surveillance, Epidemiology, and End Results Program [Internet]. Oral cavity and pharynx cancer long-term trends in SEER ageadjusted incidence rates, 1975-2017 [cited 2020 Oct 13]. Available from: https://seer.cancer.gov/explorer/ application.html?site=3\&data_type $=1 \&$ graph_type $=1 \&$ compareBy=sex\&chk_sex_3=3\&chk_sex_2=2\&race= $1 \&$ age_range $=1 \&$ hdn_stage $=101 \&$ rate_type $=1 \&$ advopt_ precision $=1 \&$ advopt_display $=2$.

2. Van Dyne EA, Henley SJ, Saraiya M, Thomas CC, Markowitz LE, Benard VB. Trends in human papillomavirus-associated cancers-United States, 19992015. MMWR 2018;67:918-24.

3. Lassen P, Lacas B, Pignon J-P, et al. Prognostic impact of HPV-associated p16-expression and smoking status on outcomes following radiotherapy for oropharyngeal cancer: The MARCH-HPV project. Radiother Oncol J Eur Soc Ther Radiol Oncol 2018;126:107-15.

4. Marur S, Forastiere AA. Head and neck squamous cell carcinoma: update on epidemiology, diagnosis, and treatment. Mayo Clin Proc 2016;91:386-96.

5. Gillison ML, Chaturvedi AK, Anderson WF, Fakhry C. Epidemiology of human papillomaviruspositive head and neck squamous cell carcinoma. JCO 2015;33:3235-42.

6. Mourad M, Jetmore T, Jategaonkar AA, Moubayed S, Moshier E, Urken ML. Epidemiological trends of head and neck cancer in the United States: a SEER population study. J Oral Maxillofac Surg 2017;75:2562-72.

7. Mehanna $H$, Beech $T$, Nicholson $T$, et al. Prevalence of human papillomavirus in oropharyngeal and nonoropharyngeal head and neck cancer-systematic review and meta-analysis of trends by time and region. Head Neck 2013;35: 747-55.

8. Moore KA, Mehta V. The growing epidemic of HPV-positive oropharyngeal carcinoma: a clinical review for primary care providers. J Am Board Fam Med 2015;28:498-503.

9. Chaturvedi AK, Engels EA, Pfeiffer RM, et al. Human papillomavirus and rising oropharyngeal cancer incidence in the United States. JCO 2011;29:4294-301.

10. Gillison ML, D'Souza G, Westra W, et al. Distinct risk factor profiles for human papillomavirus type 16-positive and human papillomavirus type 16-negative head and neck cancers. J Natl Cancer Inst 2008;100:407-20.

11. Gillison ML, Alemany L, Snijders PJF, et al. Human papillomavirus and diseases of the upper airway: head and neck cancer and respiratory papillomatosis. Vaccine 2012;30:F34-54.

12. Timbang MR, Sim MW, Bewley AF, Farwell DG, Mantravadi A, Moore MG. HPV-related oropharyngeal cancer: a review on burden of the disease and opportunities for prevention and early detection. Hum Vaccines Immunother 2019;15:1920-8.

13. Budu VA, Decuseară T, Balica NC, et al. The role of HPV infection in oropharyngeal cancer. Romanian J Morphol Embryol Rev Roum Morphol Embryol 2019;60:769-73.

14. Khalid MB, Ting P, Pai A, et al. Initial presentation of human papillomavirus-related head and neck cancer: a retrospective review. Laryngoscope 2019;129:877-82.

15. Georgopoulos R, Liu JC. Examination of the patient with head and neck cancer. Surg Oncol Clin N Am 2015;24:409-21.

16. GARDASIL9 (Human Papillomavirus 9-valent Vaccine, Recombinant) [package insert]. Whitehouse Station, NJ; Merck \& Co., Inc; revised August 2020.

17. Centers for Disease Control and Prevention [Internet]. Recommended child and adolescent 
immunization schedule for ages 18 years or younger, United States, 2020 [cited 2020 Oct 13]. Available from: https://www.cdc.gov/vaccines/ schedules/hcp/imz/child-adolescent.html\#note-hpv.

18. Centers for Disease Control and Prevention [Internet]. HPV vaccine recommendations. 2020 [cited 2020 Oct 14]. Available from: https://www. cdc.gov/vaccines/vpd/hpv/hcp/recommendations.html.

19. Centers for Disease Control and Prevention [Internet]. Human papillomavirus vaccination for adults: updated recommendations of the Advisory Committee on Immunization Practices. 2019 [cited 2020 Oct 14]. Available from: https://www.cdc.gov/ mmwr/volumes/68/wr/mm6832a3.htm.

20. Mehta V, Holmes S, Master A, Leblanc B, Caldito LG, Bocchini J. Knowledge of HPV-related oropharyngeal cancer and use of human papillomavirus vaccines by pediatricians in Louisiana. J La State Med Soc 2017;169:37-42.

21. Elam-Evans LD, Yankey D, Singleton JA, et al. National, regional, state, and selected local area vaccination coverage among adolescents aged 1317 years-United States, 2019. MMWR 2020;69: 1109-16.

22. Giuliano AR, Isaacs-Soriano $\mathrm{K}$, Torres BN, et al. Immunogenicity and safety of Gardasil among midadult aged men (27-45 years) — the MAM Study. Vaccine 2015;33:5640-6.

23. Gupta A, Perkins RB, Ortega G, Feldman S, Villa A. Barrier use during oro-genital sex and oral human papillomavirus prevalence: analysis of NHANES 2009-2014. Oral Dis 2019;25:609-16.
24. Holway GV, Hernandez SM. Oral sex and condom use in a U.S. national sample of adolescents and young adults. J Adolesc Health 2018;62:402-10.

25. D'Souza G, Fakhry C, Sugar EA, et al. Six-month natural history of oral versus cervical human papillomavirus infection. Int J Cancer 2007;121:143-50. =.

26. Golusiński W, Golusińska-Kardach E. Current role of surgery in the management of. oropharyngeal cancer. Front Oncol 2019;9:388.

27. Lim GC, Holsinger FC, Li RJ. Transoral endoscopic head and neck surgery: the contemporary treatment of head and neck cancer. Hematol Oncol Clin North Am 2015;29:1075-92.

28. Achim V, Bolognone RK, Palmer AD, et al. Longterm functional and quality-of-life outcomes after transoral robotic surgery in patients with oropharyngeal cancer. JAMA Otolaryngol- Head Neck Surg 2018;144:18-27.

29. Sethia R, Yumusakhuylu AC, Ozbay I, et al. Quality of life outcomes of transoral robotic surgery with or without adjuvant therapy for oropharyngeal cancer: QOL outcomes of TORS for oropharyngeal cancer. Laryngoscope 2018;128:403-11.

30. Castellano A, Sharma A. Systematic review of validated quality of life and swallow outcomes after transoral robotic surgery. Otolaryngol Head Neck Surg 2019;161:561-7.

31. Nichols AC, Theurer J, Prisman E, et al. Radiotherapy versus transoral robotic surgery and neck dissection for oropharyngeal squamous cell carcinoma (ORATOR): an open-label, phase 2, randomised trial. Lancet Oncol 2019;20:1349-59. 